\title{
Right Versus Left Colon Cancer Biology: Integrating the Consensus Molecular Subtypes
}

\author{
Michael S. Lee, MDa; David G. Menter, $\mathrm{PhD}^{\mathrm{b}}$; and Scott Kopetz, MD, PhD
}

\begin{abstract}
Although clinical management of colon cancer generally has not accounted for the primary tumor site, left-sided and right-sided colon cancers harbor different clinical and biologic characteristics. Right-sided colon cancers are more likely to have genome-wide hypermethylation via the $\mathrm{CpG}$ island methylator phenotype (CIMP), hypermutated state via microsatellite instability, and $B R A F$ mutation. There are also differential exposures to potential carcinogenic toxins and microbiota in the right and left colon. Gene expression analyses further shed light on distinct biologic subtypes of colorectal cancers (CRCs), with 4 consensus molecular subtypes (CMSs) identified. Importantly, these subtypes are differentially distributed between right- and left-sided CRCs, with greater proportions of the "microsatellite unstable/ immune" CMS1 and the "metabolic" CMS3 subtypes found in right-sided colon cancers. This review summarizes important biologic distinctions between right- and left-sided CRCs that likely impact prognosis and may predict for differential responses to biologic therapy. Given the inferior prognosis of stage III-IV right-sided CRCs and emerging data suggesting that anti-epidermal growth factor receptor antibody therapy is associated with worse survival in right-sided stage IV CRCs compared with left-sided cancers, these biologic differences between right- and left-sided CRCs provide critical context and may provide opportunities to personalize therapy.
\end{abstract}

J Natl Compr Canc Netw 2017;15(3):411-419

Colorectal cancer (CRC) is the fourth most commonly diagnosed cancer and the second leading cause of cancer death in the United States, ${ }^{1}$ with heterogeneous outcomes and diverse underlying pathobiologic and molecular characteristics. Accordingly, epidemiologic and histologic differences in proximal and distal CRCs have been observed: patients with right-sided CRCs are more likely to be women, be older, and have mucinous, undifferentiated, or signet-ring cell histology compared with those with left-sided CRCs. ${ }^{2-5}$ There are also different frequencies of metastasis to different organs, with rightsided CRCs more likely to metastasize to the peritoneum and left-sided CRCs more likely to metastasize

From the aDivision of Hematology/Oncology, University of North Carolina at Chapel Hill, Chapel Hill, North Carolina; and 'Department of Gastrointestinal Medical Oncology, University of Texas MD Anderson Cancer Center, Houston, Texas.

Submitted October 26, 2016; accepted for publication February 6, 2017.

The authors have disclosed that they have no financial interests, arrangements, affiliations, or commercial interests with the to the thorax or, less commonly, bone. ${ }^{6}$ The primary tumor site likely serves as a surrogate for underlying biology, including differential pathways of carcinogenesis and varying molecular features. ${ }^{7}$ With the availability of genomic platforms capable of broadly surveying gene expression and methylation, as evidenced by The Cancer Genome Atlas, ${ }^{8}$ we can now identify genomic subtypes of CRCs that are also differentially distributed between proximal and distal CRCs. This review describes the key molecular and genomic differences between proximal (right-sided) and distal (left-sided) CRCs, shedding light on the heterogeneity of clinical outcomes observed between right- and left-sided CRCs.

manufacturers of any products discussed in this article or their competitors. Dr. Kopetz has receiving funding from University of Texas MD Anderson Moon Shot Program, R01 CA 172670, and R01 CA184843. Dr. Lee has received funding from K12 CA 120780.

Correspondence: Michael S. Lee, MD, University of North Carolina at Chapel Hill, 170 Manning Drive, CB7305, Chapel Hill, NC 27599. E-mail: Michael_s_lee@med.unc.edu 


\section{Definition of Right- and Left-Sided CRC}

Notably, a consistent definition of the dividing point between right- and left-sided CRCs is not uniformly used. The most common distinction defines cancers proximal to the splenic flexure as right-sided and cancers at or distal to the splenic flexure as leftsided. ${ }^{7,9,10}$ This cutpoint is often used because most (proximal two-thirds) of the transverse colon arises embryologically from the midgut, and only the distal one-third arises from the hindgut. Although the midgut also gives rise to other organs, including most of the small bowel and the vermiform appendix, ${ }^{11}$ the rare carcinomas arising from these organs have unique biologies with different rates of key driver mutations and are clinically treated distinctly, ${ }^{12,13}$ and thus have not been included in most prior studies of CRC. Vascular supply has also been proposed as a defining characteristic of embryologic originthe superior and inferior mesenteric arteries supply the midgut and hindgut, respectively. However, this information is not routinely recorded during colonic resection. For practical purposes, given the mixed embryologic origin of the transverse colon, some studies exclude the transverse colon from analyses dichotomizing right and left CRCs.,14 Rectal cancer is also often excluded, ${ }^{2,10,15}$ particularly because localized stage II-III rectal cancers have a different treatment paradigm than colon cancers. However, biologically, based on somatic copy number variation and mRNA and microRNA expression analyses, rectal and colon cancers are indistinguishable. ${ }^{8}$ Moreover, systemic therapies for metastatic rectal and colon cancers are identical, and several recent studies have included both in their analyses. ${ }^{16,17}$ Indeed, as the frequencies of several important molecular features vary in a gradual continuum spatially from rectum through cecum, ${ }^{18}$ dichotomizing the large bowel into right- and left-sided is overly simplistic but does provide an easily captured clinical variable that connotes differing underlying biology, which is described in this review.

\section{Differences in Normal Right and Left Colon}

\section{Distinct Embryologic Etiologies}

As described earlier, distinct embryologic origins exist for the right and left sides of the colon. These distinct origins and the processes of cell migration and differentiation required during normal embryologic development require distinct gene expression patterns within the midgut and hindgut. Notably, there are differential gradients of various homeobox (HOX) and other genes establishing craniocaudal polarization. ${ }^{4,11}$ Furthermore, the rostrocaudal colonization of the intestine by enteric neuron precursors is influenced by specific protein expression, along with the associated topography and biological responses. ${ }^{19}$ Therefore, the distinct embryologic underpinnings of right and left colon likely contribute to differences in the underlying biology.

\section{Distinct Environmental Milieus}

Distinct Microbiota: The colon houses a rich microbiome of intestinal bacteria, and the presence of several bacterial strains, including Fusobacterium nucleatum, ${ }^{20,21}$ enterotoxigenic Bacteroides fragilis, ${ }^{22}$ and Enterococcus faecalis, ${ }^{23}$ is associated with CRC development. Only slight gradations of differences in the mucosa-associated microbial population distribution in different parts of the colon have been observed, ${ }^{24,25}$ indicating that within one individual the colonic microbiome is relatively uniform at different sites along the normal colon. However, there is a marked difference in the mucosal microbiota between patients who develop right-versus left-sided CRCs, ${ }^{26}$ including in the presence of bacterial biofilms, defined as mucin layers with admixed bacteria on the luminal surface of the colonic epithelium. Invasive bacterial biofilms were found in $89 \%$ of right-sided CRCs (proximal to the hepatic flexure) but in only $12 \%$ of left-sided CRCs (distal to the hepatic flexure). ${ }^{27}$ Patients who had biofilms on their tumors nearly all had similar invasive biofilms overlying normal colon tissue, even distant from the tumor. These biofilms were associated with significant decreases in epithelial E-cadherin and increased interleukin- 6 and activated Stat3, along with increased proliferation. ${ }^{27}$ Presence of these biofilms was also associated specifically with increased levels of the pro-proliferative polyamine metabolite $N^{1}, N^{12}$-diacetylspermine, indicating a unique symbiosis between the bacterial biofilm and the host cancer. ${ }^{28}$ Thus, the organization of colonic microbes into invasive biofilms, with concomitant procarcinogenic epithelial responses, has been hypothesized to contribute to 
the development of right-sided CRCs. Although additional work is required, this unique microbiome-associated pathobiology may provide a novel biomarker or a rationale for therapies to modulate the microbiome or target the metabolic or inflammatory pathways that appear to be significant features of these biofilm-associated CRCs.

Differential Bile Acid Levels: The luminal level of bile acids and their metabolites, which are putatively carcinogenic, varies with colonic location and is modulated by microbial enzymatic reactions. Bile acids are normally synthesized and conjugated with glycine and taurine residues by hepatocytes and secreted in bile. These conjugated primary bile acids undergo enzymatic deconjugation, followed by conversion to secondary bile acids such as deoxycholic acid (DCA) by anaerobic colonic microbes. Secondary bile acids are passively reabsorbed through the colonic epithelium, where they cause DNA damage through reactive oxygen and nitrogen species. ${ }^{29}$ Epidemiologic studies correlated elevated fecal bile acid concentration with increased CRC incidence, ${ }^{30,31}$ and increased DCA consumption in mice induces an increased number of colon cancers. ${ }^{32}$ Notably, there is a $>10$-fold greater concentration of the primary bile acid conjugated cholic acid in the right colon compared with the left colon, and there is greater DCA-forming enzymatic activity in cecal aspirates than in rectal fecal samples. ${ }^{33}$ The differential concentrations of bile acids in the right and left colon may therefore contribute to differential mechanisms of carcinogenesis.

\section{Differential Gene Expression and Methylation in Normal Right and Left Colon}

There are also differential gene expression profiles between normal right and left colon epithelium. Normal right colon has higher expression of cytochrome P450 family genes than the left colon, suggesting differential exposure to metabolites of ingested substances. ${ }^{4,34}$ Similarly, there are significant differences in patterns of gene methylation between the right and left colon. ${ }^{35}$ Notably, the prevalence of promoter methylation of the mismatch repair gene hMLH1 and the O-6-methylguanine-DNA methyltransferase MGMT is significantly greater in normal right colon mucosa, especially in older women, ${ }^{36}$ suggesting epigenetic aberrations in preneoplastic right colon mucosa that may be reflected in subsequent right-sided adenocarcinoma biology.

\section{Premalignant Tumors Vary by Site}

Reflecting the different predisposing factors, premalignant lesions leading to CRC vary substantially between the right and left colon. Sessile serrated adenomas are more prevalent in the right colon. Conversely, conventional tubular and tubulovillous adenomas are more uniformly distributed throughout the colon, although they are more likely to have high-grade dysplasia or associated adenocarcinoma at smaller sizes in the right colon. ${ }^{37}$ These adenomas have distinct molecular features and likely serve as precursors of biologically distinct types of CRCs (Table 1). Related partly to the differing biology, physical characteristics, and technical features, colonoscopies and polypectomies are associated with a significant decrease in the prevalence of subsequent left-sided CRCs but not right-sided CRCs. ${ }^{38,39}$

\section{Biologic Characteristics of Colon Cancers That Vary by Side}

\section{CIMP, Microsatellite Instability, and Chromosomal Instability}

Alternative paths of CRC carcinogenesis that are significantly correlated with either right- or leftsided CRCs have been identified. CRCs that have widespread genome-wide hypermethylation causing epigenetic gene silencing, termed CIMP-high, tend to be mutually exclusive from chromosomal instability tumors, defined by marked aneuploidy. ${ }^{40,41}$ CIMP. high CRCs are particularly enriched in tumors with microsatellite instability (MSI)-high due to epigen-

\begin{tabular}{|c|c|c|c|c|c|}
\hline & $\begin{array}{l}\text { CIMP- } \\
\text { High }\end{array}$ & $\begin{array}{l}\text { MSI- } \\
\text { High }\end{array}$ & $\begin{array}{c}\text { MLH1 } \\
\text { Methylation }\end{array}$ & $\begin{array}{c}\text { BRAF } \\
\text { Mutation }\end{array}$ & CIN \\
\hline \multicolumn{6}{|c|}{ Preneoplastic lesions } \\
\hline $\begin{array}{l}\text { Sessile } \\
\text { serrated } \\
\text { adenoma } \\
\text { (right-sided) }\end{array}$ & + & $+1-$ & $+1-$ & + & - \\
\hline $\begin{array}{l}\text { Conventional } \\
\text { adenoma } \\
\text { (right and } \\
\text { left-sided) }\end{array}$ & I & - & - & - & + \\
\hline \multicolumn{6}{|c|}{ Colorectal cancers } \\
\hline $\begin{array}{l}\text { Right-sided } \\
\text { CRC }\end{array}$ & $\begin{array}{l}\text { High } \\
\text { prevalence }\end{array}$ & $\begin{array}{l}\text { High } \\
\text { prevalence }\end{array}$ & $\begin{array}{l}\text { High } \\
\text { prevalence }\end{array}$ & $\begin{array}{l}\text { High } \\
\text { prevalence }\end{array}$ & $\begin{array}{l}\text { Low } \\
\text { prevalence }\end{array}$ \\
\hline $\begin{array}{l}\text { Left-sided } \\
\text { CRC }\end{array}$ & $\begin{array}{l}\text { Low } \\
\text { prevalence }\end{array}$ & $\begin{array}{l}\text { Low } \\
\text { prevalence }\end{array}$ & $\begin{array}{l}\text { Low } \\
\text { prevalence }\end{array}$ & $\begin{array}{l}\text { Low } \\
\text { prevalence }\end{array}$ & $\begin{array}{l}\text { High } \\
\text { prevalence }\end{array}$ \\
\hline
\end{tabular}

Abbreviations: CIMP, CpG island methylator phenotype; CIN, chromosomal instability; CRC, colorectal cancer; MSI, microsatellite instability. 
Lee et al

etic silencing of the MLH1 mismatch repair gene. ${ }^{41-43}$ These CIMP-high/MSI-high CRCs are more likely to be right-sided, $, 43,44$ and tumors with chromosomal instability are more likely to be left-sided. ${ }^{4,8}$

CIMP and MSI are prognostic and thus contribute to the clinical differences between right- and left-sided CRCs. CIMP-high CRCs are associated with worse survival, ${ }^{45-47}$ particularly in the $50 \%$ to $60 \%$ of CIMP-high cancers that are microsatellite stable (MSS). ${ }^{48}$ The prognostic impact of MSI depends on stage. In stage II and III cancers, MSI-high tumors have superior prognosis compared with MSS tumors. ${ }^{49-51}$ Conversely, though only $4 \%$ of stage IV CRCs are MSI-high, ${ }^{52}$ these tumors have historically been associated with inferior survival. ${ }^{53}$ Notably, this inferior prognosis compared with MSS CRCs remained significant in the BRAF wild-type subgroup, although there was a uniformly poor prognosis in BRAF-mutant tumors that was not significantly altered by microsatellite status. ${ }^{53}$ However, given emerging evidence for the efficacy of immune checkpoint antibodies, such as anti-programmed death-1 (PD-1), in the treatment of MSI-high CRC, ${ }^{54}$ the prognosis associated with MSI in stage IV disease may soon drastically change. Nevertheless, given the enrichment of CIMP-high and MSI-high cancers in right-sided CRCs and their known prognostic impact, these factors likely contribute to historical differential outcomes by side.

\section{Mutation Profiles}

There are different rates of mutations in key oncogenes and tumor suppressors between right- and leftsided CRCs (Table 2). ${ }^{8}$ Notably, mutations in BRAF V600E, which are associated with significantly inferior survival in stage IV CRC, ${ }^{53,55,56}$ are significantly more common in right-sided CRCs. ${ }^{57}$ Conversely, mutations in APC and TP53 are enriched in leftsided CRCs. ${ }^{8}$ Recently, different patterns of mutations in APC, TP53, and KRAS were identified as conferring differential prognoses in CRC, ${ }^{58}$ although given the differences in mutation frequency by site, additional analysis via right or left primary site is warranted. Besides point mutations, potentially targetable amplifications of receptor tyrosine kinases, such as ERBB2 and epidermal growth factor receptor (EGFR), are also more common in left-sided CRCs. ${ }^{4}$ These underlying variations in mutation and genomic patterns may explain differential outcomes

\begin{tabular}{|c|c|c|c|}
\hline & $\begin{array}{c}\text { Total } \\
(\mathrm{n}=276)\end{array}$ & $\begin{array}{l}\text { Righta }^{a} \\
(n=92)\end{array}$ & $\begin{array}{c}\text { Left }^{\mathrm{a}} \\
(\mathrm{n}=161)\end{array}$ \\
\hline$A P C$ & $75.0 \%$ & $63.6 \%$ & $81.9 \%$ \\
\hline TP53 & $54.0 \%$ & $34.8 \%$ & $64.6 \%$ \\
\hline KRAS & $42.0 \%$ & $45.5 \%$ & $40.3 \%$ \\
\hline PIKЗCA & $20.1 \%$ & $27.3 \%$ & $14.6 \%$ \\
\hline$F B X W 7$ & $16.5 \%$ & $22.7 \%$ & $12.5 \%$ \\
\hline SMAD4 & $11.6 \%$ & $15.2 \%$ & $9.7 \%$ \\
\hline TGFBR2 & $10.3 \%$ & $27.3 \%$ & $1.4 \%$ \\
\hline BRAF & $9.4 \%$ & $24.2 \%$ & $2.1 \%$ \\
\hline NRAS & $8.9 \%$ & $7.6 \%$ & $9.0 \%$ \\
\hline
\end{tabular}

${ }^{\text {aExcludes }} 18$ transverse colon and 5 unknown.

to therapy or offer potential new therapies for which site would be an important consideration.

\section{Gene Expression Profiles}

Several studies have identified CRC subtypes by performing unsupervised gene expression clustering, with several consistent features across all subtyping schemes, such as MSI-high/CIMP-high subtype predominantly in right-sided CRCs and a mesenchymal subtype with poor prognosis. However, there was a lack of consistency among other subtypes delineated, and each classification scheme had between 3 and 6 distinct subtypes identified. ${ }^{59-64}$ To unite these disparate subtyping schemes, an international CRC Subtyping Consortium applied multiple independent subtyping classifications across a unified set of 3,962 samples. The association between these disparate classification schemes was determined and 4 consensus molecular subtypes (CMSs) emerged. ${ }^{65}$

The CMSs: Key features of the 4 CMSs are outlined in Table 3. Notably, CMS1, which is predominantly composed of right-sided CRCs, is also enriched for MSI-high, CIMP-high, and BRAF mutation. Although genotypic features such as KRAS and BRAF mutation status are enriched in some subtypes (ie, BRAF in CMS1; KRAS in CMS3), their presence or absence does not specifically define any subtype, demonstrating the limited value of genotype in defining broader CRC biology. Similarly, there is heterogeneity among right-sided CRCs, such that even though right-sided CRCs are enriched in CMS1 


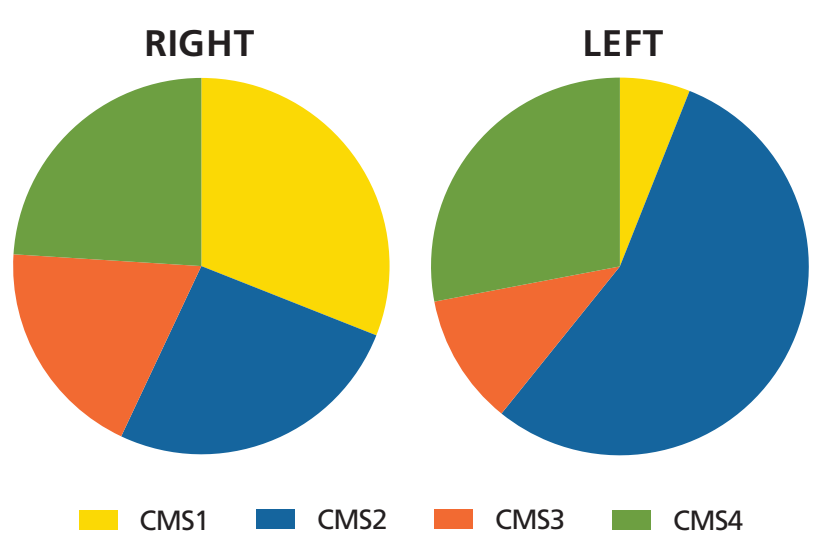

Figure 1. Distribution of CMS by right- and left-sided CRC. ${ }^{65}$ Right-side CRC was defined as the cecum through transverse colon, and left-side CRC was defined as the splenic flexure through rectum. Abbreviations: CMS, consensus molecular subtype; CRC, colorectal cancer.

and CMS3, all 4 CMSs are represented among right-sided $\mathrm{CRCs}^{65}$ (Figure 1).

The CMSs are associated with prognosis, which varies based on relapse status. CMS4 has the worst overall survival (OS) among all stages and the worst relapse-free survival among those who were initially stages I-III. However, among those who experienced relapse, OS was markedly worse in CMS1 and superior in CMS2. For example, in the PETACC-3 trial, the hazard ratio (HR) for CMS1 versus CMS2 for survival after relapse was 5.00 (95\% CI, 2.86-9.09)..$^{65}$

The underlying unique biology of each subtype is also reflected by differential activation of various biologic pathways (Table 3). CMS1 has increased immune infiltration signatures, which are expected given the association between mutation burden, neoantigen load, and immune infiltration in CRCs. ${ }^{66}$ CMS2 has marked activation of WNT and MYC pathways. CMS3 has marked activation of multiple metabolic pathways. Finally, CMS4 is enriched for signatures of epithelial-mesenchymal transition (EMT), the pro-EMT transforming growth factor $-\beta{ }^{67}$ and angiogenic pathways. CMS4 is also characterized by particularly high expression of stroma-derived genes associated with cancer-associated fibroblasts. ${ }^{68,69}$ Although this does introduce the potential for misclassifying tumors if tumor microdissection is not performed and greater stromal contamination results, ${ }^{70}$ a similar poor prognostic mesenchymal subtype was reproducibly independently identified in several classification schemes us- ing microdissected tumors, suggesting that CMS4 is a bona fide subtype with a more prominent stromal microenvironment that helps drive tumor biology. ${ }^{71}$

EREG and AREG Expression: The EGFR ligands epiregulin (EREG) and amphiregulin (AREG) are differentially expressed between right-and left-sided CRCs. High tumor expression of EREG and AREG is associated with greater response rates and improved outcomes with anti-EGFR antibody therapy in patients with KRAS and NRAS wild-type metastatic CRCs. ${ }^{72-74}$ EREG and AREG expressions are significantly higher in left-sided CRCs, ${ }^{4,44,75}$ and are inversely correlated with promoter methylation and CIMP-high status. ${ }^{44}$ Although the association of EREG and AREG expression and improved outcomes with anti-EGFR inhibitors has been highlighted as evidence of autocrine/juxtacrine EGFR signaling driving CRC growth, the strong association of sidedness and EREG/AREG expression suggests that part of the effect may be confounded by other differences in CRC biology.

\section{CRC Side and Clinical and Prognostic Impact}

\section{Stage II-III Disease}

There is differential prognosis by stage between patients with right- and left-sided CRCs. Tumor registries and retrospective cohorts suggest that right-sided tumors have a slightly better prognosis in stage II colon cancer, but slightly worse prognosis in stage III disease, likely associated with the higher prevalence of good-prognosis MSI-high tumors in right-sided stage II cancers. ${ }^{2,9,10}$ Moreover, analyses of prospective clinical trials of patients with stage III CRC who received adjuvant chemotherapy also demonstrated inferior disease-free survival in those with right-sided CRCs (HR, 0.70; 95\% CI, 0.61-0.81). ${ }^{76}$

\section{Stage IV Disease}

Patients with metastatic CRC with a right-sided primary also have inferior prognosis compared with those with a left-sided primary. ${ }^{17}$ This is highlighted by a pooled analysis of 3 studies of 2,027 evaluable patients treated with first-line chemotherapy, in which those with left-sided CRCs had significantly better progression-free survival (PFS) and OS compared with those with right-sided CRCs, including after adjusting for BRAF mutation and mucinous histology. ${ }^{77}$ 
Lee et al

\begin{tabular}{|c|c|c|c|c|}
\hline & $\begin{array}{c}\text { CMS1 } \\
\text { MSI/Immune }\end{array}$ & $\begin{array}{c}\text { CMS2 } \\
\text { Canonical }\end{array}$ & $\begin{array}{c}\text { CMS3 } \\
\text { Metabolic }\end{array}$ & $\begin{array}{c}\text { CMS4 } \\
\text { Mesenchymal }\end{array}$ \\
\hline Proportion of total samples & $14 \%$ & $37 \%$ & $13 \%$ & $23 \%$ \\
\hline$\%$ right-sided (vs left-sided) & $77 \%$ & $23 \%$ & $51 \%$ & $35 \%$ \\
\hline Grade 3 (vs 1-2) & $45 \%$ & $5 \%$ & $12 \%$ & $19 \%$ \\
\hline MSI-high & $76 \%$ & $1 \%$ & $16 \%$ & $8 \%$ \\
\hline CIMP-high & $67 \%$ & $3 \%$ & $16 \%$ & $10 \%$ \\
\hline Hypermutated ( $\geq 180$ events) & $94 \%$ & $6 \%$ & $28 \%$ & $9 \%$ \\
\hline $\begin{array}{l}\text { Somatic copy number } \\
\text { alteration-high }\end{array}$ & $20 \%$ & $92 \%$ & $54 \%$ & $84 \%$ \\
\hline KRAS mutated & $23 \%$ & $28 \%$ & $68 \%$ & $38 \%$ \\
\hline NRAS mutated & $4 \%$ & $7 \%$ & $9 \%$ & $4 \%$ \\
\hline$B R A F$ mutated & $42 \%$ & $1 \%$ & $7 \%$ & $7 \%$ \\
\hline \multirow[t]{5}{*}{ Gene set enrichment analysis } & Immune infiltration & Epithelial signature & Epithelial signature & $\begin{array}{l}\text { Epithelial mesenchymal } \\
\text { transition }\end{array}$ \\
\hline & $\begin{array}{l}\text { Cytotoxic T cell } \\
\text { infiltration }\end{array}$ & MYC activation & $\begin{array}{l}\text { Sugar/amino acid/ } \\
\text { nucleotide metabolism }\end{array}$ & $\begin{array}{l}\text { transition } \\
\text { Matrix remodeling }\end{array}$ \\
\hline & $T_{H} 1$ infiltration & SRC activation & \multirow{3}{*}{$\begin{array}{l}\text { Fructose/mannose } \\
\text { metabolism } \\
\text { Glutamine metabolism }\end{array}$} & Stromal infiltration \\
\hline & PD-1 activation & & & TGF- $\beta$ activation \\
\hline & $\begin{array}{l}\text { Natural killer cell } \\
\text { infiltration }\end{array}$ & & & VEGF-VEGFR activation \\
\hline
\end{tabular}

Abbreviations: CIMP, CpG island methylator phenotype; CMS, consensus molecular subtypes; MSI, microsatellite instability; TH1, type 1 helper T cell; PD-1, programmed death-1; TGF- $\beta$, transforming growth factor- $\beta$; VEGF, vascular endothelial growth factor; VEGFR, VEGF receptor. Data from Guinney J, Dienstmann R, Wang X, et al. The consensus molecular subtypes of colorectal cancer. Nat Med 2015;21:1350-1356.

Primary CRC site is prognostic but not predictive of outcome with bevacizumab-based therapy. An analysis of 2 prospective randomized controlled trials of chemotherapy with or without bevacizumab found that a statistical interaction test between side and bevacizumab use was nonsignificant, indicating that side is not a predictive biomarker for or against benefit of bevacizumab. ${ }^{77}$

However, primary CRC site is both prognostic and predictive of benefit with anti-EGFR therapy among patients with KRAS wild-type, refractory, metastatic CRC. In the CO.17 randomized trial, among those with KRAS codon 12/13 wild-type disease, there was a significant improvement in PFS with cetuximab in patients with a left-sided primary (HR, 0.28; 95\% CI, 0.18-0.45), whereas there was no difference in PFS among those with a right-sided primary (HR, 0.73; 95\% CI, 0.42-1.27; interaction $P=.002) .{ }^{15}$ Additional retrospective studies also showed that patients with left-sided CRCs had better PFS with anti-EGFR therapy compared with those with right-sided CRCs, even among patients with KRAS/BRAF wild-type ${ }^{4}$ and extended RAS and BRAF wild-type mutations. ${ }^{78}$
Similar results have been found with first-line cetuximab combined with chemotherapy among patients with CRC wild-type in KRAS and NRAS exons 2 to 4 (pan-RAS wild-type). In the CALGB/ SWOG 80405 study randomizing patients with KRAS wild-type metastatic CRC to receive first-line chemotherapy with either cetuximab or bevacizumab, 474 patients were known to be pan-RAS wildtype. In this study, a right-sided primary was associated with inferior OS among the cetuximab group (HR, 1.81 vs left-sided; 95\% CI, 1.27-2.56). PFS was also significantly worse among those with a rightsided primary who received cetuximab, but was not significantly worse among those who received bevacizumab, ${ }^{16}$ thus indicating that a right-sided primary is a predictive biomarker of inferior survival with cetuximab-based chemoimmunotherapy (interaction $P=.0009$ for OS). ${ }^{16}$ These results are corroborated by an analysis of the 394 patients with pan-RAS wildtype metastatic CRC randomized to receive first-line FOLFIRI with either cetuximab or bevacizumab in the FIRE-3 study. In this trial, there was again inferior OS with a right-sided primary, markedly among patients who received cetuximab (median OS, 18.3 vs 38.3 months; $P<.001$ ), but also among 


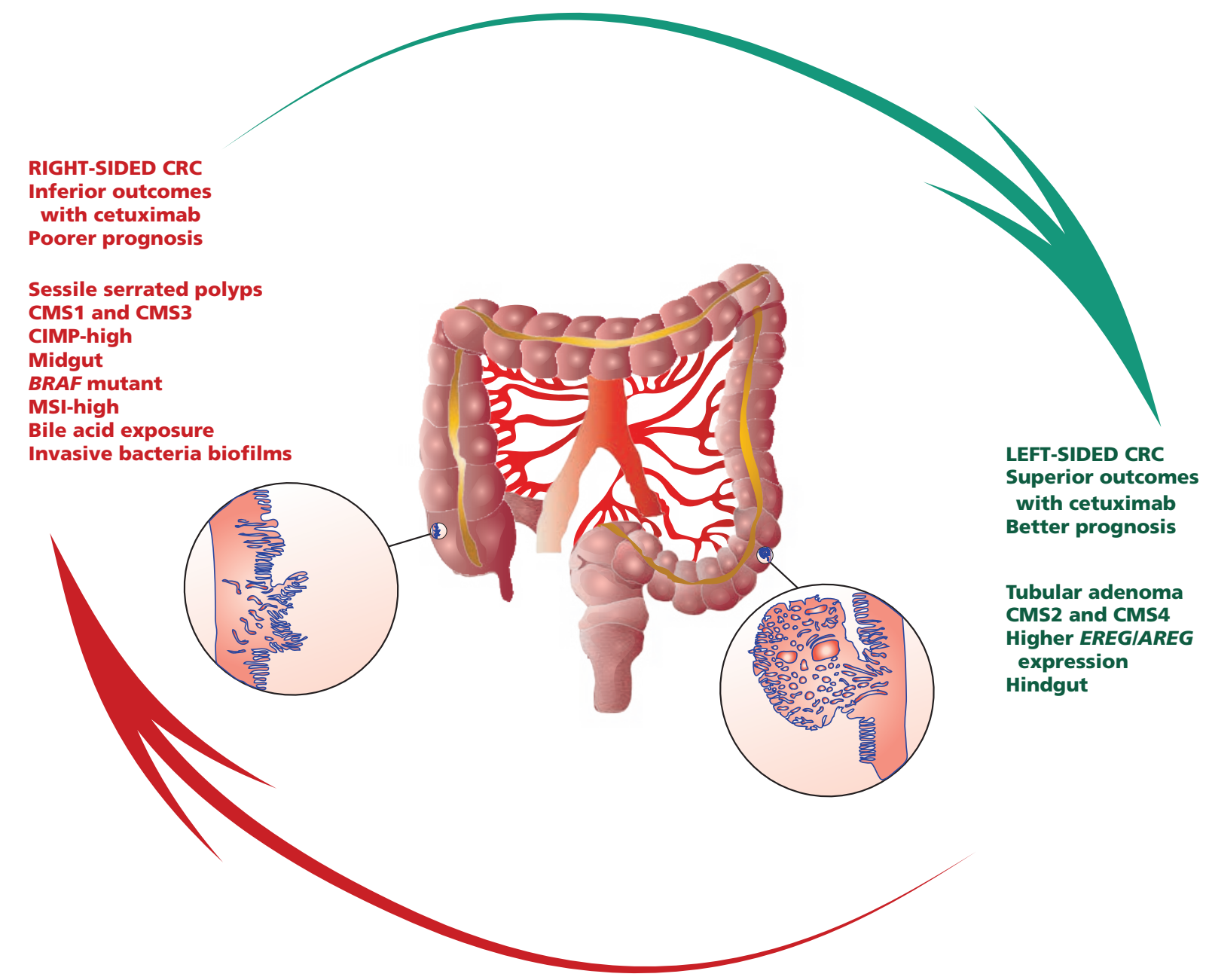

Figure 2. Summary of key biologic differences between right- and left-sided CRCs.

Abbreviations: AREG, amphiregulin; CIMP, CpG island methylator phenotype; CMS, consensus molecular subtype; CRCs, colorectal cancers; EREG, epiregulin; MSI, microsatellite instability.

those who received bevacizumab (median OS, 23.0 vs 28.0 months; $P=.04) .16,79$ On multivariate analysis, including BRAF-mutant status, a significant difference was seen in OS between treatment and tumor location $^{79}$ (interaction $P=.0015$ ). Finally, in the CRYSTAL study, 364 patients with pan-RAS wildtype metastatic CRC were randomized to receive first-line FOLFIRI with or without cetuximab, and OS was again significantly inferior in patients with right-sided tumors compared with left-sided tumors who received cetuximab and FOLFIRI (median OS, 18.5 vs 28.7 months; HR, 1.93; 95\% CI, 1.24-2.99)..$^{79}$ On multivariate analysis, including BRAF-mutant status, there was again a significant interaction $P$ value of 0.0241 for OS between treatment and tumor location. ${ }^{79}$ However, information on other molecular features, including characteristics of the BRAF-mutant cohorts, was not available for these studies, and there may well be enrichment for BRAF-mutant status among the population of KRAS wild-type rightsided CRCs that may confound the data. Additional data including subsequent lines of therapy by side may also provide insight into reasons for differential survival observed in these trials. Given the strikingly limited OS observed among patients with RAS wildtype right-sided CRC treated with first-line antiEGFR therapy, consensus has been emerging that patients with right-sided CRC should not receive first-line anti-EGFR-based chemoimmunotherapy. 
Lee et al

\section{Conclusions}

Right- and left-sided CRCs have markedly different underlying biologic characteristics (Figure 2), with enrichment for MSI-high, CIMP-high, and BRAFmutant cancers among right-sided CRCs. These characteristics are most notably included among the CMS1 genomic subtype, which is also enriched among right-sided CRCs, although CMS3 also skews toward right-sided CRCs. Differential distribution of these genomic CRC subtypes and other biologic features among right- and left-sided CRCs may contribute to the inferior prognosis of advanced-stage right-sided CRCs and an inferior outcome with antiEGFR therapy in right-sided CRC. Future clinical trials in CRC will need to consider important sideassociated variables and side itself when considering outcomes, and hopefully will allow for personalized therapy based on underlying biologic vulnerabilities.

\section{References}

1. Howlader N, Noone A, Krapcho M, et al. SEER Cancer Statistics Review, 1975-2011. Vol. 2014. Bethesda, MD: National Cancer Institute; 2014

2. Benedix F, Kube R, Meyer F, et al. Comparison of 17,641 patients with rightand left-sided colon cancer: differences in epidemiology, perioperative course, histology, and survival. Dis Colon Rectum 2010;53:57-64.

3. Gonzalez EC, Roetzheim RG, Ferrante JM, Campbell R. Predictors of proximal vs. distal colorectal cancers. Dis Colon Rectum 2001;44:251-258.

4. Missiaglia E, Jacobs B, D'Ario G, et al. Distal and proximal colon cancers differ in terms of molecular, pathological, and clinical features. Ann Oncol 2014;25:1995-2001.

5. Bufill JA. Colorectal cancer: evidence for distinct genetic categories based on proximal or distal tumor location. Ann Intern Med 1990;113:779-788.

6. Riihimaki M, Hemminki A, Sundquist J, Hemminki K. Patterns of metastasis in colon and rectal cancer. Sci Rep 2016;6:29765.

7. Iacopetta B. Are there two sides to colorectal cancer? Int J Cancer 2002;101:403-408.

8. The Cancer Genome Atlas Network. Comprehensive molecular characterization of human colon and rectal cancer. Nature 2012;487:330 337.

9. Meguid RA, Slidell MB, Wolfgang CL, et al. Is there a difference in survival between right- versus left-sided colon cancers? Ann Surg Oncol 2008;15:2388-2394

10. Weiss JM, Pfau PR, O'Connor ES, et al. Mortality by stage for right- versus left-sided colon cancer: analysis of surveillance, epidemiology, and end results-Medicare data. J Clin Oncol 2011;29:4401-4409.

11. Schoenwolf GC, Bleyl SB, Brauer PR, Francis-West PH. Larsen's Human Embryology, 5th edition. Philadelphia, PA: Churchill Livingstone; 2015.

12. Raghav KP, Shetty AV, Kazmi SM, et al. Impact of molecular alterations and targeted therapy in appendiceal adenocarcinomas. Oncologist 2013;18:1270-1277.

13. Raghav K, Overman MJ. Small bowel adenocarcinomas-existing evidence and evolving paradigms. Nat Rev Clin Oncol 2013;10:534-544.

14. Venook AP, Niedzwiecki $D$, Innocenti F, et al. Impact of primary $\left(1^{\circ}\right)$ tumor location on overall survival (OS) and progression-free survival (PFS) in patients (pts) with metastatic colorectal cancer (mCRC): analysis of CALGB/SWOG 80405 (Alliance) [abstract]. J Clin Oncol 2016;34(Suppl):Abstract 3504.

15. Brule SY, Jonker DJ, Karapetis CS, et al. Location of colon cancer (rightsided versus left-sided) as a prognostic factor and a predictor of benefit from cetuximab in NCIC CO.17. Eur J Cancer 2015;51:1405-1414.

16. Venook A, Niedzwiecki D, Ou F-S, et al. Impact of primary tumor location on overall survival and progression free survival in patients with metastatic colorectal cancer: analysis of all RAS wt subgroup on CALGB/SWOG 80405 (Alliance). Presented at the European Society for Medical Oncology 2016 Congress; October 7-11, 2016; Copenhagen, Denmark.

17. Schrag D, Weng S, Brooks G, et al. The relationship between primary tumor sidedness and prognosis in colorectal cancer [abstract]. J Clin Oncol 2016;34(Suppl):Abstract 3505.

18. Yamauchi M, Morikawa T, Kuchiba A, et al. Assessment of colorectal cancer molecular features along bowel subsites challenges the conception of distinct dichotomy of proximal versus distal colorectum. Gut 2012;61:847-854.

19. Fu M, Tam PK, Sham MH, Lui VC. Embryonic development of the ganglion plexuses and the concentric layer structure of human gut: a topographical study. Anat Embryol (Berl) 2004;208:33-41.
20. Castellarin M, Warren RL, Freeman JD, et al. Fusobacterium nucleatum infection is prevalent in human colorectal carcinoma. Genome Res 2012;22:299-306

21. Kostic AD, Gevers D, Pedamallu CS, et al. Genomic analysis identifies association of Fusobacterium with colorectal carcinoma. Genome Res 2012;22:292-298.

22. Toprak NU, Yagci A, Gulluoglu BM, et al. A possible role of Bacteroides fragilis enterotoxin in the aetiology of colorectal cancer. Clin Microbiol Infect 2006;12:782-786

23. Winters MD, Schlinke TL, Joyce WA, et al. Prospective case-cohort study of intestinal colonization with enterococci that produce extracellular superoxide and the risk for colorectal adenomas or cancer. Am J Gastroenterol 1998;93:2491-2500

24. Lepage $P$, Seksik $P$, Sutren $M$, et al. Biodiversity of the mucosa-associated microbiota is stable along the distal digestive tract in healthy individuals and patients with IBD. Inflamm Bowel Dis 2005;11:473-480.

25. Sears CL, Garrett WS. Microbes, microbiota, and colon cancer. Cell Host Microbe 2014;15:317-328.

26. Flemer B, Lynch DB, Brown JM, et al. Tumour-associated and non-tumourassociated microbiota in colorectal cancer [published online ahead of print March 18, 2016]. Gut 2016; doi: 10.1136/gutjnl-2015-309595.

27. Dejea CM, Wick EC, Hechenbleikner EM, et al. Microbiota organization is a distinct feature of proximal colorectal cancers. Proc Natl Acad Sci U S A 2014;111:18321-18326.

28. Johnson CH, Dejea CM, Edler D, et al. Metabolism links bacterial biofilms and colon carcinogenesis. Cell Metab 2015;21:891-897.

29. Venturi M, Hambly RJ, Glinghammar B, et al. Genotoxic activity in human faecal water and the role of bile acids: a study using the alkaline comet assay. Carcinogenesis 1997;18:2353-2359.

30. Reddy BS, Hedges AR, Laakso K, Wynder EL. Metabolic epidemiology of large bowel cancer: fecal bulk and constituents of high-risk North American and low-risk Finnish population. Cancer 1978;42:2832-2838.

31. Hill MJ, Taylor AJ, Thompson MH, Wait R. Fecal steroids and urinary volatile phenols in four Scandinavian populations. Nutr Cancer 1982;4:67-73.

32. Bernstein $\mathrm{C}$, Holubec $\mathrm{H}$, Bhattacharyya AK, et al. Carcinogenicity of deoxycholate, a secondary bile acid. Arch Toxicol 2011;85:863-871.

33. Thomas LA, Veysey MJ, French G, et al. Bile acid metabolism by fresh human colonic contents: a comparison of caecal versus faecal samples. Gut 2001;49:835-842.

34. Glebov OK, Rodriguez LM, Nakahara K, et al. Distinguishing right from left colon by the pattern of gene expression. Cancer Epidemiol Biomarkers Prev 2003;12:755-762.

35. Kaz AM, Wong CJ, Dzieciatkowski S, et al. Patterns of DNA methylation in the normal colon vary by anatomical location, gender, and age. Epigenetics 2014;9:492-502.

36. Menigatti M, Truninger $\mathrm{K}$, Gebbers JO, et al. Normal colorectal mucosa exhibits sex- and segment-specific susceptibility to DNA methylation at the hMLH1 and MGMT promoters. Oncogene 2009;28:899-909.

37. Gupta S, Balasubramanian BA, Fu T, et al. Polyps with advanced neoplasia are smaller in the right than in the left colon: implications for colorecta cancer screening. Clin Gastroenterol Hepatol 2012;10:1395-1401, e1392.

38. Nishihara R, Wu K, Lochhead $\mathrm{P}$, et al. Long-term colorectal-cancer incidence and mortality after lower endoscopy. N Engl J Med 2013;369:1095-1105. 
Right Versus Left Colon Cancer Biology

39. Brenner $\mathrm{H}$, Hoffmeister M, Arndt V, et al. Protection from right- and leftsided colorectal neoplasms after colonoscopy: population-based study. J Natl Cancer Inst 2010;102:89-95.

40. Cheng YW, Pincas $\mathrm{H}$, Bacolod MD, et al. $\mathrm{CpG}$ island methylator phenotype associates with low-degree chromosomal abnormalities in colorectal cancer. Clin Cancer Res 2008;14:6005-6013.

41. Goel A, Nagasaka $T$, Arnold $C N$, et al. The $\mathrm{CpG}$ island methylator phenotype and chromosomal instability are inversely correlated in sporadic colorectal cancer. Gastroenterology 2007;132:127-138.

42. Kane MF, Loda M, Gaida GM, et al. Methylation of the hMLH1 promoter correlates with lack of expression of hMLH1 in sporadic colon tumors and mismatch repair-defective human tumor cell lines. Cancer Res 1997;57:808-811.

43. Toyota M, Ahuja N, Ohe-Toyota M, et al. CpG island methylator phenotype in colorectal cancer. Proc Natl Acad Sci U S A 1999;96:86818686.

44. Lee MS, McGuffey EJ, Morris JS, et al. Association of CpG island methylator phenotype and EREG/AREG methylation and expression in colorectal cancer. Br J Cancer 2016;114:1352-1361.

45. Shen L, Catalano PJ, Benson AB III, et al. Association between DNA methylation and shortened survival in patients with advanced colorectal cancer treated with 5-fluorouracil based chemotherapy. Clin Cancer Res 2007;13:6093-6098

46. Juo YY, Johnston FM, Zhang DY, et al. Prognostic value of $\mathrm{CpG}$ island methylator phenotype among colorectal cancer patients: a systematic review and meta-analysis. Ann Oncol 2014;25:2314-2327.

47. Cha Y, Kim KJ, Han SW, et al. Adverse prognostic impact of the CpG island methylator phenotype in metastatic colorectal cancer. Br J Cancer 2016;115:164-171.

48. Dahlin AM, Palmqvist R, Henriksson ML, et al. The role of the CpG island methylator phenotype in colorectal cancer prognosis depends on microsatellite instability screening status. Clin Cancer Res 2010;16:18451855.

49. Samowitz WS, Curtin K, Ma KN, et al. Microsatellite instability in sporadic colon cancer is associated with an improved prognosis at the population level. Cancer Epidemiol Biomarkers Prev 2001;10:917-923.

50. Benatti P, Gafa R, Barana D, et al. Microsatellite instability and colorectal cancer prognosis. Clin Cancer Res 2005;11:8332-8340.

51. Popat S, Hubner R, Houlston RS. Systematic review of microsatellite instability and colorectal cancer prognosis. J Clin Oncol 2005;23:609-618.

52. Koopman M, Kortman GA, Mekenkamp L, et al. Deficient mismatch repair system in patients with sporadic advanced colorectal cancer. $\mathrm{Br}$ J Cancer 2009; 100:266-273

53. Tran B, Kopetz S, Tie J, et al. Impact of BRAF mutation and microsatellite instability on the pattern of metastatic spread and prognosis in metastatic colorectal cancer. Cancer 2011;117:4623-4632.

54. Le DT, Uram JN, Wang H, et al. PD-1 blockade in tumors with mismatchrepair deficiency. N Engl J Med 2015;372:2509-2520.

55. Lochhead P, Kuchiba A, Imamura Y, et al. Microsatellite instability and BRAF mutation testing in colorectal cancer prognostication. J Nat Cancer Inst 2013;105:1151-1156.

56. Tol J, Nagtegaal ID, Punt CJ. BRAF mutation in metastatic colorectal cancer. N Engl J Med 2009;361:98-99.

57. Gonsalves WI, Mahoney MR, Sargent DJ, et al. Patient and tumor characteristics and BRAF and KRAS mutations in colon cancer, NCCTG/ Alliance N0147 [published corrections appears in J Natl Cancer Inst 2014;106:dju228]. J Natl Cancer Inst 2014;106:dju106.

58. Schell MJ, Yang M, Teer JK, et al. A multigene mutation classification of 468 colorectal cancers reveals a prognostic role for APC. Nat Commun 2016;7:11743

59. Budinska E, Popovici V, Tejpar S, et al. Gene expression patterns unveil a new level of molecular heterogeneity in colorectal cancer. J Pathol 2013;231:63-76.
60. De Sousa E Melo F, Wang X, Jansen M, et al. Poor-prognosis colon cancer is defined by a molecularly distinct subtype and develops from serrated precursor lesions. Nat Med 2013;19:614-618.

61. Marisa L, de Reynies A, Duval A, et al. Gene expression classification of colon cancer into molecular subtypes: characterization, validation, and prognostic value. PLoS Med 2013;10:e1001453.

62. Roepman P, Schlicker A, Tabernero J, et al. Colorectal cancer intrinsic subtypes predict chemotherapy benefit, deficient mismatch repair and epithelial-to-mesenchymal transition. Int J Cancer 2014;134:552-562.

63. Sadanandam A, Lyssiotis CA, Homicsko K, et al. A colorectal cancer classification system that associates cellular phenotype and responses to therapy. Nat Med 2013;19:619-625.

64. Schlicker A, Beran G, Chresta CM, et al. Subtypes of primary colorectal tumors correlate with response to targeted treatment in colorectal cell lines. BMC Med Genomics 2012;5:66

65. Guinney J, Dienstmann R, Wang X, et al. The consensus molecular subtypes of colorectal cancer. Nat Med 2015;21:1350-1356

66. Giannakis M, Mu XJ, Shukla SA, et al. Genomic correlates of immune-cell infiltrates in colorectal carcinoma. Cell Rep 2016;17:1206.

67. $\mathrm{Xu}$ J, Lamouille S, Derynck R. TGF-beta-induced epithelial to mesenchymal transition. Cell Res 2009;19:156-172.

68. Isella C, Terrasi A, Bellomo SE, et al. Stromal contribution to the colorectal cancer transcriptome. Nat Genet 2015;47:312-319.

69. Calon A, Lonardo E, Berenguer-Llergo A, et al. Stromal gene expression defines poor-prognosis subtypes in colorectal cancer. Nat Genet 2015;47:320-329.

70. Dunne PD, McArt DG, Bradley CA, et al. Challenging the cancer molecular stratification dogma: intratumoral heterogeneity undermines consensus molecular subtypes and potential diagnostic value in colorectal cancer. Clin Cancer Res 2016;22:4095-4104.

71. Morris JS, Kopetz S. Tumor microenvironment in gene signatures: critical biology or confounding noise? Clin Cancer Res 2016;22:3989-3991.

72. Khambata-Ford S, Garrett CR, Meropol NJ, et al. Expression of epiregulin and amphiregulin and K-ras mutation status predict disease control in metastatic colorectal cancer patients treated with cetuximab. J Clin Oncol 2007;25:3230-3237.

73. Jacobs B, De Roock W, Piessevaux H, et al. Amphiregulin and epiregulin mRNA expression in primary tumors predicts outcome in metastatic colorectal cancer treated with cetuximab. J Clin Oncol 2009;27:5068-5074.

74. Seligmann JF, Elliott F, Richman SD, et al. Combined epiregulin and amphiregulin expression levels as a predictive biomarker for panitumumab therapy benefit or lack of benefit in patients with RAS wild-type advanced colorectal cancer [published online ahead of print February 11, 2016]. JAMA Oncol, doi: 10.1001/jamaoncol.2015.6065.

75. Adams RA, Fisher D, Farragher S, et al. Use of epiregulin (EREG) and amphiregulin (AREG) gene expression to predict response to cetuximab (cet) in combination with oxaliplatin $(\mathrm{Ox})$ and $5 \mathrm{FU}$ in the first-line treatment of advanced colorectal cancer (aCRC) [abstract]. J Clin Oncol 2012;30(Suppl):Abstract 32.

76. Sinicrope FA, Mahoney MR, Smyrk TC, et al. Prognostic impact of deficient DNA mismatch repair in patients with stage III colon cancer from a randomized trial of FOLFOX-based adjuvant chemotherapy. J Clin Oncol 2013;31:3664-3672

77. Loupakis F, Yang D, Yau L, et al. Primary tumor location as a prognostic factor in metastatic colorectal cancer. J Natl Cancer Inst 2015;107:dju427.

78. Moretto R, Cremolini C, Rossini D, et al. Location of primary tumor and benefit from anti-epidermal growth factor receptor monoclonal antibodies in patients with RAS and BRAF wild-type metastatic colorectal cancer. Oncologist 2016;21:988-994.

79. Tejpar S, Stintzing S, Ciardiello F, et al. Prognostic and predictive relevance of primary tumor location in patients with RAS wild-type metastatic colorectal cancer: retrospective analyses of the CRYSTAL and FIRE-3 trials [published online ahead of print October 10, 2016]. JAMA Oncol, doi:10.1001/jamaoncol.2016.3797. 